\title{
ARTICLE OPEN \\ Microlayered flow structure around an acoustically levitated droplet under a phase-change process
}

\author{
Koji Hasegawa ${ }^{1}$, Yutaka $\mathrm{Abe}^{2}$ and Atsushi Goda ${ }^{3}$
}

\begin{abstract}
The acoustic levitation method (ALM) has found extensive applications in the fields of materials science, analytical chemistry, and biomedicine. This paper describes an experimental investigation of a levitated droplet in a 19.4-kHz single-axis acoustic levitator. We used water, ethanol, water/ethanol mixture, and hexane as test samples to investigate the effect of saturated vapor pressure on the flow field and evaporation process using a high-speed camera. In the case of ethanol, water/ethanol mixtures with initial ethanol fractions of 50 and $70 \mathrm{wt} \%$, and hexane droplets, microlayered toroidal vortexes are generated in the vicinity of the droplet interface. Experimental results indicate the presence of two stages in the evaporation process of ethanol and binary mixture droplets for ethanol content $>10 \%$. The internal and external flow fields of the acoustically levitated droplet of pure and binary mixtures are clearly observed. The binary mixture of the levitated droplet shows the interaction between the configurations of the internal and external flow fields of the droplet and the concentration of the volatile fluid. Our findings can contribute to the further development of existing theoretical prediction.
\end{abstract}

npj Microgravity (2016) 2, 16004; doi:10.1038/npjmgrav.2016.4; published online 10 March 2016

\section{INTRODUCTION}

Because of its simplicity and usefulness as a tool for non-contact manipulation, the acoustic levitation method (ALM) has for decades been a focus of investigation by researchers in the fields of material science, analytical chemistry, and biomedical industry. ${ }^{1-6}$ Although several contactless handling methods have been investigated in the last decade, ${ }^{7,8}$ ALM has been shown to be the best technique for microassembly., ${ }^{9,10}$ The microassembly of chemical and biological components is currently of great importance in the biomedical industry. Classical processing techniques used for chemical and biological components cannot be used for microassembly, because the usually neglected surface forces can dominate the adhesion between the component and gripper. $^{2-4}$ The levitation method is a promising alternative for treating adhesion due to surface forces. In addition, ALM is an alternative method for the preparation of the nano-to-pico volume samples for processing without contamination from walls, other external objects, or other samples. ${ }^{9,10}$

Acoustic streaming is known to be caused by acoustic waves. $^{11,12}$ Such acoustic streaming can be classified into two types. The first is caused by spatial attenuation, which generally arises owing to compressibility (i.e., Eckart streaming). The second is owing to the strong nonlinear behavior at interfaces (i.e., Schlichting streaming), and can occur even if the fluid is considered incompressible; e.g., a vibrating sphere in an otherwise quiescent liquid. ${ }^{13,14}$ Using undercooling processing in a study of the effect of acoustic streaming on molten liquid materials, Chung et al. $^{15}$ reported that acoustic streaming possibly affects an obstacle in space by changing the internal motion of a droplet. In an experimental investigation of the external streaming flow around an acoustically levitated droplet, Trinh et al. ${ }^{16}$ have qualitatively observed the external flow and revealed toroidal vortices generated around the droplet. Using computational techniques, Rednikov et al. ${ }^{17-19}$ provided an explanation of the flow phenomena observed by Trinh et al. ${ }^{16}$ Because the model by Rednikov et al. ${ }^{17}$ was an idealization of the experimental conditions, their computational results ${ }^{17}$ did not agree with the experimental results obtained by Trinh et al. ${ }^{16}$ To understand the mechanism of the external flow and its influence on the internal circulation of an acoustically levitated droplet, a threedimensional observation of the internal flow is required. Zhao et al. ${ }^{20,21}$ numerically investigated the relation between the internal and external flows and suggested that the recirculation in the Stokes layer should be generated in order to explain the flow fields inside and outside the droplet. Yarin et al. ${ }^{22}$ theoretically studied the formulation of streaming flow around an acoustically levitated droplet and suggested that internal circulation may be driven by gas flow near the drop surface. The flow fields were characterized both in the absence and presence of the sample in a levitator. On the bais of their previous work, ${ }^{22}$ Yarin et al. $^{23}$ also developed a theoretical model of an acoustically driven droplet of the evaporation of binary mixtures. The authors experimentally revealed that the configurations of the internal and external flows are influenced by the viscosity of the levitated droplet. $^{24-27}$

The nonlinear and dynamic behavior of an acoustically levitated droplet may affect its heat transfer, mass transport, and solidification properties. As mentioned above, many investigations have been conducted over the past thirty years to exploit the potential of the ALM. Although many analytical techniques for investigating the flow fields around a droplet have been developed, ${ }^{17-23}$ there have been only few experimental findings. Although perfect sample manipulation with ALM has been highly expected recently, this has not been achieved partly because of

\footnotetext{
${ }^{1}$ Department of Mechanical Engineering, Kogakuin University, Tokyo, Japan; ${ }^{2}$ Division of Engineering Mechanics and Energy,Faculty of Engineering, Information and Systems, University of Tsukuba, Tsukuba, Japan and ${ }^{3}$ Department of Engineering, Mechanics and Energy, University of Tsukuba, Tsukuba, Japan.

Correspondence: K Hasegawa (kojihasegawa@cc.kogakuin.ac.jp)

Received 27 May 2015; revised 2 December 2015; accepted 14 December 2015
} 
thermofluid nonlinearity. ${ }^{28-30}$ Determining the nonlinear mechanisms and elucidating the phenomena in acoustically levitated droplets are crucial. This study aims to investigate the external flow structure and evaporation process of an acoustically levitated droplet experimentally and quantitatively.

\section{RESULTS}

The authors presented the flow fields around water and ethanol droplets in the previous work. $^{28}$ Although we successfully visualized the flow field of an acoustically droplet based on the Particle image velocimetry (PIV) results, an in-depth measurement
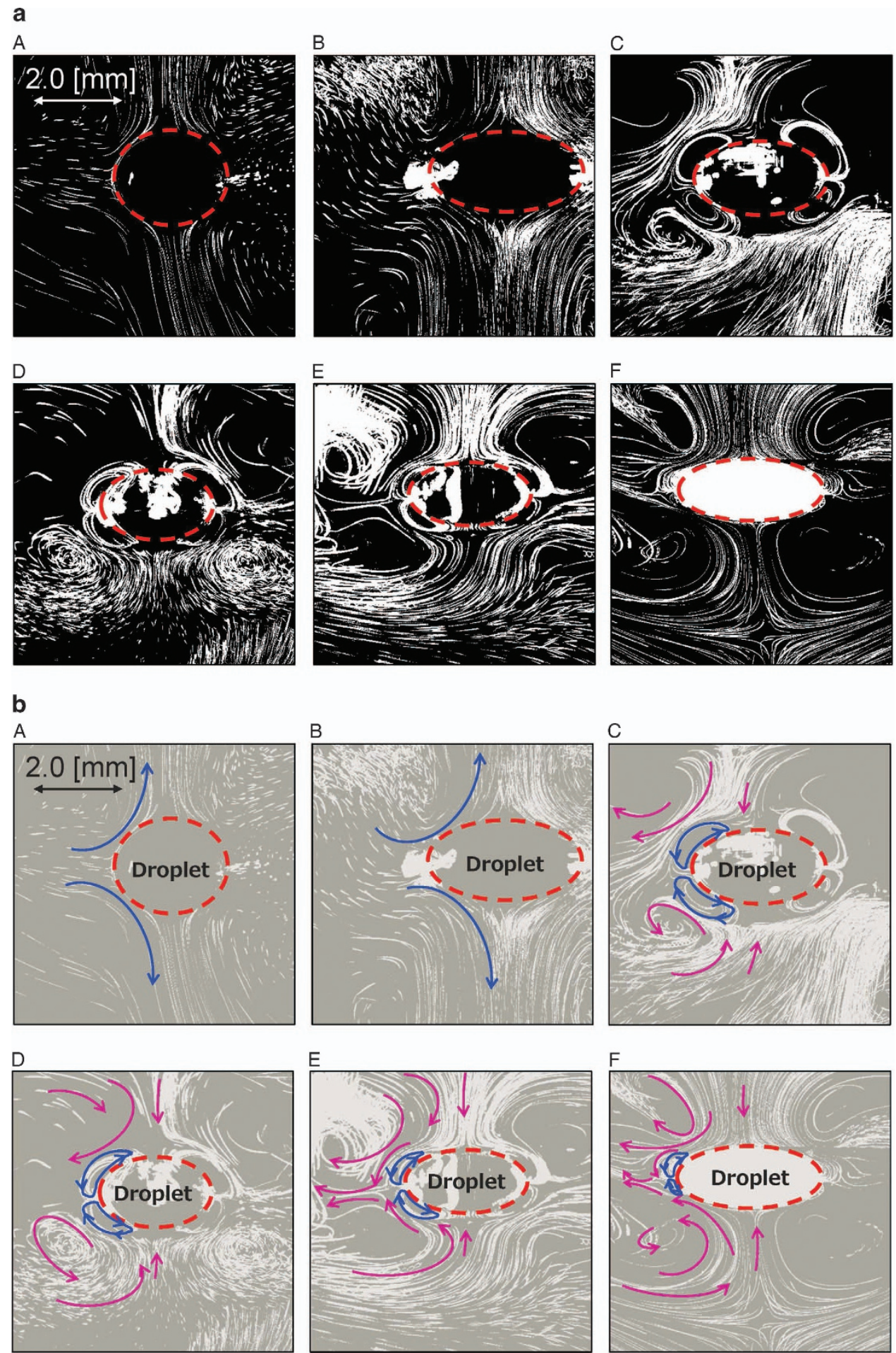

Figure 1. (a) Flow fields in the vicinity of the droplet interface: $A$, water; ethanol solution (B, 25 wt $\%$; $C, 50$ wt $\%$; and $D, 75$ wt $\%$ ethanol); $E$, ethanol; and F, hexane. (b) The flow direction corresponding to a: A, water; ethanol solution (B, 25 wt $\%$; $C, 50$ wt $\%$; and $D, 75$ wt $\%$ ethanol); $\mathrm{E}$, ethanol; and $\mathrm{F}$, hexane. 
on the flow field in the vicinity of the levitated droplet is required with volatile droplets by trajectory analysis.

Figure 1a shows the flow field in the vicinity of the droplet interface. The white line is the trajectory of the particles obtained by multiexposure for $0.1 \mathrm{~s}$. The red dashed line shows the droplet interface. Water, ethanol solution (25, 50, and 75 wt\% ethanol), ethanol, ${ }^{29}$ and hexane ${ }^{29}$ were used in our experiments. For the droplets consisting of 50 and 75 wt\% ethanol water/ethanol mixtures, pure ethanol, and hexane, a microlayered vortex was observed in the vicinity of the droplet. Conversely, for the droplets consisting of water and 25 wt $\%$ water/ethanol mixture, the microlayered vortexes were not observed. Figure $1 \mathrm{~b}$ shows the flow direction corresponding to Figure 1a. The circulating vortex flows in from the sides of the droplet and is directed into the top and bottom of the droplet along the interface. Then, before reaching the top and bottom ends, it is circulated back to the sides. The flow direction is consistent with the flow behavior expected in the case of a non-volatile fluid.

As shown in Figure 2, as the concentration of ethanol in the droplet increases, the size of the circular vortex $\delta$ decreases. The size of the circular vortex that is generated around the hexane droplet is smaller than that of the ethanol droplet. These results imply that a microlayered vortex in the vicinity of the droplet was affected by saturated vapor pressure.

Figure 3 shows the evaporation of water, ${ }^{28}$ ethanol, ${ }^{28}$ and water/ethanol mixtures with an initial ethanol fraction of $10,50,{ }^{28}$ and $70 \mathrm{wt} \%$ as a function of time. Initial experimental conditions are listed in Table 1. An inspection of Figure 3 shows that two stages are present in the evaporation process of ethanol and of binary mixture droplets for ethanol content $>10 \%$ as indicated by the nonlinear time dependence of evaporation. The time of the first stage increased with the increase of the initial ethanol fraction. This means that the evaporation process is dominated by a more volatile liquid at the initial stage, and the droplet contains no solute (ethanol) at the second stage. Although Yarin's

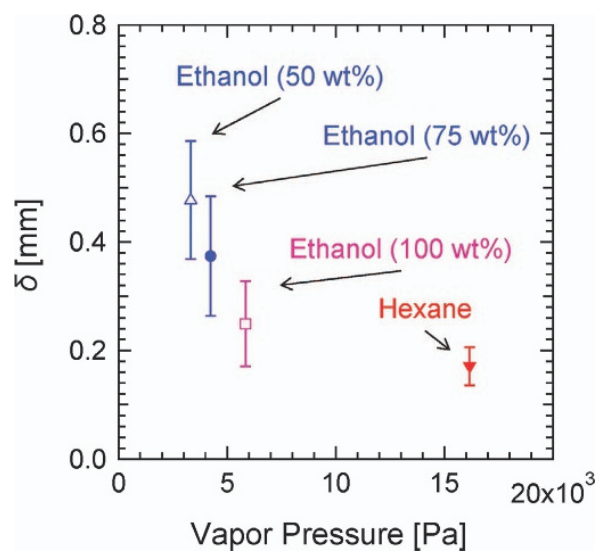

Figure 2. Effect of vapor pressure on thickness of vortex around each droplet. work uses the ventilation system on their experimental setup, ${ }^{23}$ our results are in good agreement with their experimental data without any ventilation.

Figure 4 represents the temporal evolution of the internal and external flow fields of water/ethanol mixtures with an initial ethanol fraction of $50 \mathrm{wt} \%{ }^{28}$ Average velocity vector fields of the internal flow are visualized by PIV. The external flow field is obtained by the multiply-exposed trajectory. At $t=0 \mathrm{~s}$, internal and external flow fields are the same as those of the ethanol droplet, which exhibits toroidal vortexes in and near the droplet surface. The configuration of the flow fields changes as the time progresses, and at the final stage of evaporation $(t=600 \mathrm{~s})$, internal and external flow fields are the same as those of the water droplet, which exhibits one circulation in the droplet, and no toroidal vortexes are present in the vicinity of the droplet surface.

\section{DISCUSSION}

Our results show that the toroidal vortexes (forced convection) that affect the mass transfer of the levitated droplet appeared near the droplet surface because of the higher concentration of ethanol. The Marangoni effect, which is the mass transfer along an interface between two fluids due to surface tension gradient, may be one of the causes of these phenomena.

The acoustic streaming observed in this study is induced by the theoretically predicted Stokes-layer recirculation. ${ }^{20,21}$ This recirculation drives internal and external flow fields through the droplet interface. Figure 4 indicates that the concentration of ethanol solution also affects the temporal evolution of flow configurations. It is clearly identified the internal and external flow fields of the acoustically levitated droplet of the binary mixture by visualization measurement. These experimental data can contribute to the further development of existing theoretical prediction. ${ }^{23}$ The significance of these results is that the acoustic streaming affects the tangential fluid motion on the levitated droplet surface fora

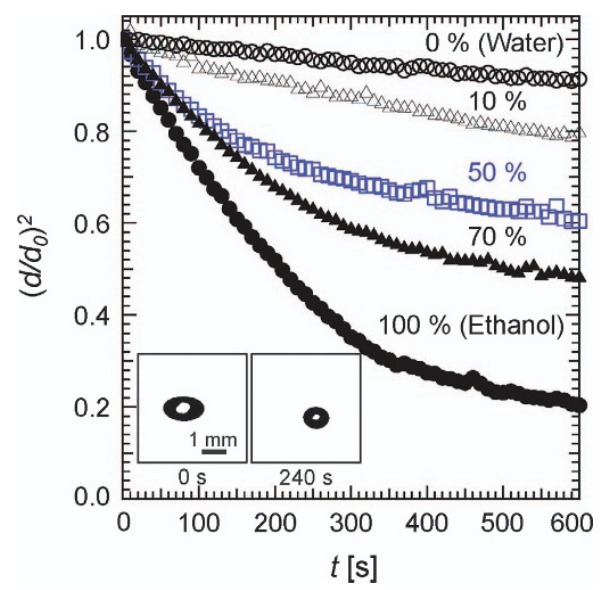

Figure 3. Evaporation of water, ethanol, and water/ethanol mixtures with an initial ethanol fraction of 10,50 , and $70 \%$.

Table 1. Initial and ambient conditions in the experiments with water, ethanol, and water/ethanol mixtures at $24 \pm 2{ }^{\circ} \mathrm{C}$

\begin{tabular}{lccc}
\hline Sample & Initial mass fraction of ethanol (\%) & Initial droplet diameter (mm) & Initial aspect ratio (-) \\
\hline Water & 0 & 4.1 & 1.9 \\
Water/Ethanol & 10 & 2.9 & 1.4 \\
Water/Ethanol & 50 & 2.4 & 2.3 \\
Water/Ethanol & 70 & 2.5 & 1.9 \\
Ethanol & 100 & 2.4 & 1.9 \\
\hline
\end{tabular}




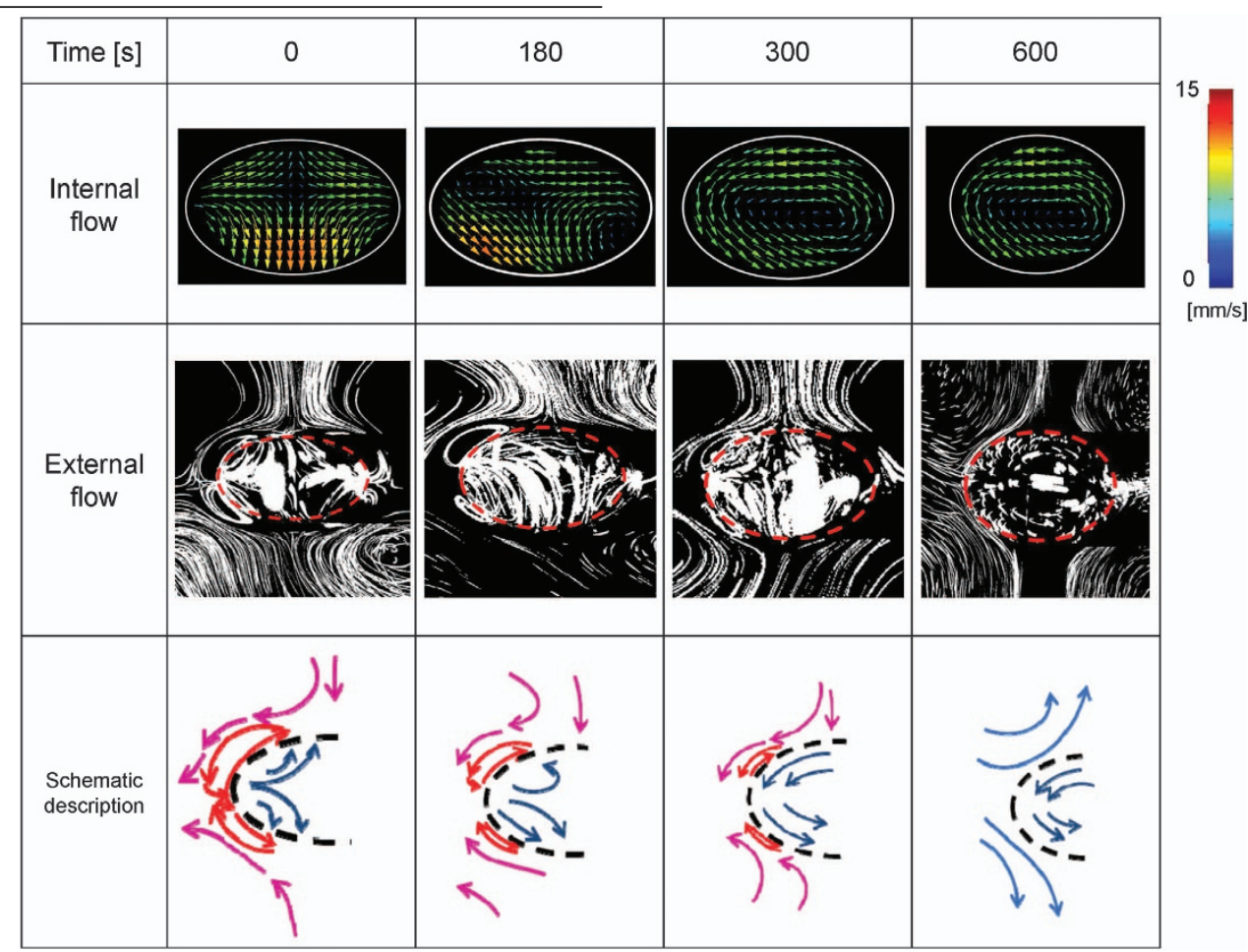

Figure 4. Temporal evolution of the internal and external flow fields of water/ethanol mixtures with an initial ethanol fraction of 50 wt\%.

higher concentration of ethanol and the hexane droplet, as well as the acoustic streaming was affected by the changes in the concentration of the surrounding fluid due to droplet evaporation.

In this study, we identified the internal and external flow fields of the acoustically levitated droplet of the binary mixture by visualization measurement. In the case of the water droplet, a toroidal vortex is generated around the bottom of the droplet. For ethanol, water/ethanol mixtures with an initial ethanol fraction of 50 and $70 \mathrm{wt} \%$, and hexane droplets, microlayered toroidal vortexes were generated in the vicinity of the droplet interface. A comparison of the results for these droplets shows that a higher saturated pressure gives rise to a microlayered vortex at the interface. The experimental results for the later stage of ethanol droplet evaporation imply that the evaporation process is dominated by the more volatile liquid (ethanol) at the initial stage and that the pure ethanol droplet is gradually transformed into a pure water droplet by preferential evaporation. An examination of the binary mixture droplet shows not only how the flow fields of an acoustically levitated droplet affect the evaporation process but also how the concentration of the volatile fluid affects the configuration of the flow fields.

\section{MATERIALS AND METHODS}

\section{Experimental setup}

Acoustic levitation is based on the production of a standing wave with equally spaced nodes and antinodes between the horns. ${ }^{7} \mathrm{~A}$ sinusoidal wave signal at a frequency of $19.4 \mathrm{kHz}$ is generated from a function generator. ${ }^{29} \mathrm{~A}$ signal-produced acoustic standing wave provides radiation pressure arising from a vertical orientation of the gap. When a droplet is manually inserted around a pressure node of the acoustic standing wave, the droplet can be levitated in air. In a gaseous host medium, typical sound pressure levels were tuned between 160 and $163 \mathrm{~dB}$ for ensuring a stable levitation of the droplet. The distance between the horn and reflector was set as $\sim 47 \mathrm{~mm}$.

To observe the external flow around a droplet, seeding water mist generated by a nebulizer was added. The size of each seeding particle is $\sim 5 \mu \mathrm{m}$ in diameter. A streaming flow field induced by a standing wave can be visualized by illuminating the entrained tracer particles. The flow patterns were recorded using a high-speed video camera (Photoron, Tokyo, Japan; FASTCAM-MAX). PIV was implemented, and a quantitative flow field was visualized using a sheet laser (Laser Quantum, Stockport, Cheshire, England, UK; excel+mpc6000), high-speed video camera, and computer. In the present study, water, ethanol, water/ethanol mixture, and hexane are used to elucidate the effect of saturated vapor pressure on the evaporation process. The evaporation processes of a volatile droplet were observed using a high-speed camera, and the changes in droplet diameter with time were derived from the snapshots.

\section{Statistical analysis}

We levitated each droplet more than 5 times and found no statistically significant differences $(P>0.05)$. The levitated droplet was recorded by a high-speed camera with the spatial resolution of $\sim 10 \mu \mathrm{m} /$ pixel so that the measurement error on the droplet diameter and the size of the circular vortex could be $<5 \%$ at a maximum.

\section{ACKNOWLEDGMENTS}

This work has been supported by JSPS KAKENHI Grant number 16360097, 18360083, and 23360081. We thank Satoshi Matsumoto, Katsuhiro Nishinari, Tadashi Watanabe, Hiroyuki Kitahata, Akiko Kaneko, and Hikaru Ishii for the insightful comments and suggestions.

\section{COMPETING INTERESTS}

The authors declare no conflict of interest.

\section{REFERENCES}

1. Brandt, E. H. Acoustic physics: suspended by sound. Nature $\mathbf{4 1 3}, 474-475$ (2001).

2. Vandaele, V., Lambert, P. \& Delchambre, A. Non-contact handling in microassembly: acoustical levitation. Precis. Eng. 29, 491-505 (2005).

3. Priego-Capote, F. \& de Castro, L. Ultrasound-assisted levitation: lab-on-a-drop. Trends Anal. Chem. 25, 856-867 (2006).

4. Scheeline, A. \& Behrens, R. L. Potential of levitated drops to serve as microreactors for biophysical measurements. Biophys. Chem. 165, 1-12 (2012).

5. Chainani, E. T., Choi, W. H., Ngo, K. T. \& Scheeline, A. Mixing in colliding, ultrasonically levitated drops. Anal. Chem. 86, 2229-2237 (2014). 
6. Santesson, S. \& Nilsson, S. Airborne chemistry: acoustic levitation in chemical analysis. Anal. Bioanal. Chem. 378, 1704-1709 (2004).

7. Yarin, A. L., Pfaffenlehner, M. \& Tropea, C. On the acoustic levitation of droplets J. Fluid Mech. 356, 65-91 (1998).

8. Foresti, D., Nabavi, M. \& Poulikakos, D. On the acoustic levitation stability behavior of spherical and ellipsoidal particles. J. Fluid Mech. 709, 581-592 (2012).

9. Foresti, D., Nabavi, M., Klingauf, M., Ferrari, A. \& Poulikakos, D. Acoustophoretic contactless transport and handling of matter in air. Proc. Natl Acad. Sci. USA 110, 12549-12554 (2013).

10. Foresti, D. \& Poulikakos, D. Acoustophoretic contactless elevation, orbital transport and spinning of matter in air. Phys. Rev. Lett. 112, 024301-1-5 (2014).

11. Riley, N. On a sphere oscillating in a viscous liquid. Quart. J. Mech. Appl. Math. 19, 461-472 (1966).

12. Riley, N. Steady streaming. Ann. Rev. of Fluid Mech. 33, 43-65 (2001).

13. Sadhal S. Microscale Acoustofluidics. 256-311 (Royal Society of Chemistry, 2014),

14. Green R., Ohlin M., Wiklund M. Microscale Acoustofluidics. 312-336 (Royal Society of Chemistry, 2014)

15. Chung, S. K. \& Trinh, E. H. Containerless protein crystal growth in rotating levitated drops. J. Cryst. Growth 194, 384-397 (1998).

16. Trinh, E. H. \& Robey, J. L. Experimental study of streaming flows associated with ultrasonic levitators. Phys. Fluids 6, 3567-3579 (1994).

17. Rednikov, A. Y. \& Riley, N. A simulation of streaming flows associated with acoustic levitators. Phys. Fluids 14, 1502-1510 (2002).

18. Rednikov, A. Y., Zhao, H. \& Sadhal, S. S. Steady streaming around a spherical drop displaced from the velocity antinode in an acoustic levitation field. Q. J. Mech. Appl. Math. 59, 377-397 (2006).

19. Rednikov, A. Y. \& Sadhal, S. S. Steady streaming from an oblate spheroid due to vibration along its axis. J. Fluid Mech. 499, 345-380 (2004).

20. Zhao, H., Sadhal, S. S. \& Trinh, E. H. Singular perturbation analysis of an acoustically levitated sphere: Flow about the velocity node. J. Acoust. Soc. Am. 106 589-595 (1999).

21. Zhao, H., Sadhal, S. S. \& Trinh, E. H. Internal circulation in a drop in an acoustic field. J. Acoust. Soc. Am. 106, 3289-3295 (1999).
22. Yarin, A. L., Brenn, G., Kastner, O., Rensink, D. \& Tropea, C. Evaporation of acoustically levitated drops. J. Fluid Mech. 399, 151-204 (1999).

23. Yarin, A. L., Brenn, G., Kastner, O. \& Rensink, D. Evaporation of acoustically levitated droplets of binary liquid mixtures. Int. J. Heat Fluid Flow 23, 471-486 (2002).

24. Abe, Y., Hyuga, D., Yamada, S. \& Aoki, K. Study on internal flow and surface deformation of large droplet levitated by ultrasonic wave. Ann. N.Y. Acad. Sci. 1077, 49-62 (2006).

25. Yamamoto, Y., Abe, Y., Fujiwara, A., Hasegawa, K. \& Aoki, K. Internal flow of acoustically levitated droplet. Microgravity Sci. Technol. 20, 277-280 (2008).

26. Abe, Y., Yamamoto, Y., Hyuga, D., Awazu, S. \& Aoki, K. Study on interfacial stability and internal flow of a droplet levitated by ultrasonic wave. Ann. N. Y. Acad. Sci. 1161, 211-224 (2009).

27. Hasegawa, K., Kaneko, A., Aoki, K. \& Abe, Y. PIV measurement of internal and external flow of an acoustically levitated droplet. Int. J. Trans. Phenomena 12, 151-160 (2011).

28. Ishii, H., Hasegawa, K., Kaneko, A. \& Abe, Y. Internal and external flow structure and mass transport phenomena of an acoustically levitated droplet. Trans. Jpn Soc. Mech. Eng. B 78, 1696-1709 (2012).

29. Goda, A., Hasegawa, K., Kaneko, A., Kanagawa, T. \& Abe, Y. External flow structure and interfacial transport phenomena of an acoustically levitated droplet. Jpn J. Multiphase Flow 28, 539-546 (2015).

30. Shitanishi, K., Hasegawa, K., Kaneko, A. \& Abe, Y. Study on Heat Transfer and Flow Characteristic Under Phase-Change Process of an Acoustically Levitated Droplet. Micrograv. Sci. Technol. 26, 305-312 (2014).

(c) (i) (2) This work is licensed under a Creative Commons AttributionNonCommercial-ShareAlike 4.0 International License. The images or other third party material in this article are included in the article's Creative Commons license, unless indicated otherwise in the credit line; if the material is not included under the Creative Commons license, users will need to obtain permission from the license holder to reproduce the material. To view a copy of this license, visit http:// creativecommons.org/licenses/by-nc-sa/4.0/ 